\title{
The Feasibility Analysis of Two-dimensional Near-infrared Spectroscopy Applied to the Donkey Meat Identification
}

\author{
Limin Shao ${ }^{1, a^{*}}$ and Dong Liang ${ }^{1, b}$ \\ ${ }^{1}$ College of Mechanical and Electrical Engineering, Hebei Agricultural University, Baoding, China \\ ashaolm@126.com, b1340913757@qq.com
}

Keywords: Two-dimensional Near-infrared Spectroscopy; Donkey Meat; Identification; Feasibility Analysis

\begin{abstract}
Donkey meat has high edible value. Baoding City donkey meat is well-known local specialties, commonly known as "sky dragon meat, underground donkey". In order to avoid illegal commercial tenants selling other meats to consumers, it should identify the meat species. Based on the near-infrared spectroscopy technology, combined with the two-dimensional correlation spectroscopy technology, two-dimensional near-infrared spectroscopy could be used to identify the donkey meat effectively. The research results are of great significance to improve the automation of livestock product identification in China, and safeguarding the legitimate rights of the consumers and donkey meat farmers.
\end{abstract}

\section{Introduction}

In the world, China is the country with the largest number of donkeys. The breeding stock is about ten million each year [1]. In recent years, with the popularization of agricultural mechanization, the donkey gradually developed from the draft animal to the meat. The protein content of donkey meat is significantly higher than that of other meats, such as pork, beef, chicken and mutton [2]. The content of fat and cholesterin is both lower than those of other meats. Meanwhile, donkey meat contains multiple amino acids and mineral elements, which are benefit the human body. In this way, donkey meat is the optimal meat food for hypertension, obesity, atherosclerotic and old peoples. Because of the high value of donkey meat, its price has continued rise in rencent years. But the numbers of domestic donkeys is decreasing year by year. Thus, the donkey meat resources are very scarce. In the pursuit of profits, some illegal commercial tenants sell beef, horse meat and mule meat, or even pork as donkey meat, seriously harming the rights and interests of consumers. At present, the identification methods of donkey meat were mainly used some chemical methods, such as polymerase chain reaction and chromogenic reaction. These methods require destructive operations on donkey meat samples, and they belong to the non-destructive testing methods. In addition, due to the high cost of pretreatment, these methods are not suitable for real-time identification of large number of donkey meat samples.

\section{Two-dimensional Near-infrared Spectroscopy}

Definition. The concept of two-dimensional correlation spectroscopy was first proposed in the field of Nuclear Magnetic Resonance analysis. Untill 1993, Noda bring forward the concept of generalized two-dimensional correlat ion spectroscopy, and the types of spectra used in two-dimensional correlation spectroscopy are extended to Raman spectrum, fluorescence spectrum and near infrared spectrum [3]. Two-dimensional near-infrared spectroscopy is the combination of two-dimensional correlation spectroscopy technology and near-infrared spectroscopy technology, correlation analysis was carried out for the near infrared spectrum of the system in the disturbed process, and obtained the two-dimensional scale information of the spectrum, including synchronous correlation spectra and asynchronous correlation spectra [4]. If applying an external disturbance to a system to be analysed, the system will generate a dynamic change, the near infrared spectrum behavior will change. Using the correlation analysis to deal with the system spectrogram, two-dimensional correlation near infrared spectrum could be obtained [5-6]. And the two-dimensional correlation near infrared spectrum has a 
strong resolving power to the overlapping signals. So, it is wide used in the research fields of the characteristic analysis and the structure analysis, and so on.

Overseas and Domestic Research Status. Two-dimensional near-infrared spectroscopy has been proposed for more than 20 years. By now, hardly any domestic and foreign researchers have used the technology to analyze agricultural products and food. But, this technology has its unique advantages in the analysis of some components of biological materials, such as protein and ascorbic acid.

In the foreign contry, Ozaki used two-dimensional correlation near-infrared spectroscopy to analyze the structural differences of ovalbumin in aqueous solutions under the thermal disturbance and serum protein deals with isomerizations with varying $\mathrm{pH}$ disturbance [7]. Liu et al. analyzed the synchronous and asynchronous correlation spectra of chicken samples under thermal perturbation using two-dimensional correlation visible near-infrared spectroscopy, it was found that the absorption intensity decreased at multiple wavelengths of 475, 520, 585, and 445 and $560 \mathrm{~nm}$, and that the C-H bond is easy to be oxidized through the asynchronous correlation spectrum [8]. In the next year, Liu and Chen studied the physical chemistry changes and biological changes of frozen chicken during thawing by using two-dimensional visible near-infrared spectroscopy [9].

Domestic scholars mainly focus on traditional Chinese medicine and edible oil using two-dimensional near-infrared spectroscopy. Liu et al. used two-dimensional correlation near-infrared spectroscopy to analyze the variation rule of the near-infrared spectra in the process of thermal disturbance, found the extended two-dimensional correlation spectra significantly different, and it could be used as the basis for rapid identification of Chinese medicinal materials, Cortex Periplocae and Cortex Acanthopanacis [10]. $\mathrm{Lu}$ et al. used two-dimensional correlation near-infrared spectroscopy to identify Fructus Lycii from four different regions under the temperature disturbance, and found that the two-dimensional near-infrared synchronous and asynchronous correlation spectra of Fructus Lycii from different regions were significantly different, and the difference was caused by different active components and content [11]. Combined with Fourier Transform near-infrared spectroscopy technology, Chen used two-dimensional correlation analysis to identify four different edible oils under the concentration disturbance, it was found that there were significant differences in the two-dimensional correlation near-infrared spectroscopy of $4220 \sim 4310 \mathrm{~cm}^{-1}$, and the results show that two-dimensional correlation near-infrared spectroscopy can effectively identify edible vegetable oil [12].

After analysis of the above research literatures, the two-dimensional near-infrared spectral analysis technology at home and abroad is still a relatively new analysis technology, using the technology of agricultural and animal products and food for effective identification. At the same time, the technology could extract the characteristic peaks and radical group absorption characteristics covered by the one-dimensional near infrared spectrum. In addition, it could contribute to understanding the internal principle of near-infrared identification, and also to provide theoretical explanation from the molecular and radical group level for the samples identification.

\section{Methodology}

The mechanism study of near-infrared spectroscopy analysis is always a difficulty in the study of near-infrared spectroscopy. In rencent years, with the development of the application of near-infrared spectroscopy technology in China, it has become a research hotspot.

In terms of donkey meat identification, our research team did a lot of preliminary research [13]. Taking the fresh donkey meat and other kinds of meat as the research objects, a high-precision donkey meat identification model using fast and lossless near-infrared spectroscopy and stoichiometry was established. The result of research showed that, the accuracy rate of meat loaf samples was $98.96 \%$, the accuracy rate of $7 \mathrm{~mm}$ diameter minced samples was $97.53 \%$, and the accuracy rate of $5 \mathrm{~mm}$ and $3 \mathrm{~mm}$ diameter minced samples were both $100 \%$.

Although it could be obtained satisfactory results by using the near-infrared spectra of samples directly for quantitative or qualitative identification, the detection mechanism is not as clear. This greatly limits the application of near-infrared spectroscopy, and it is hard to be a reliable and credible arbitration 
technique. In the meantime, the development of scientific theory of near-infrared spectroscopy analysis is greatly hindered.

The spectral information used by the research group in previous studies was indirect information that characterizes the structural absorbing radical group characteristics, extracted from near infrared spectroscopy of meat samples by computer. The information about these structural radical groups is very vague. This way, it led to the near-infrared spectroscopy becomed a "black box" technology. Just know the input of the "black box" is the spectrogram information of near-infrared spectroscopy containing the structural radical group information of meat samples, and the output of the "black box" is the classification result of different kinds of meat samples. The intermediate operation process of the "black box", that is to say, the identifing mechanism does know nothing. The analysis feature of near-infrared spectroscopy is caused by heavy absorption overlapping of near-infrared spectroscopy, and without specificity. Many chemical compounds generate absorption throughout the near-infrared spectroscopy, which made the near-infrared spectroscopy analysis more difficult. Which indicator plays a key role in the identification? Much of the messages are unclear, the corresponding structural radical group of the key indicator, the spectral information and the attibution of the radical group.

Choose the fresh donkey meat and other meat circulating in the market as the research object samples, integrated application of multidisciplinary technologies and independent innovation, such as near-infrared spectroscopy, two-dimensional correlation spectroscopy, information technology and chemometrics. Study on the identification mechanism of meat varieties based on two-dimensional correlation near-infrared spectroscopy, the research results will have important theoretical significance in the near infrared spectroscopy detection and identification of animal products.

Research technical route of the two-dimensional near-infrared spectroscopy applied to the donkey meat identification is shown in Figure.1.

\section{Conclusions}

Studying the identification mechanism of meat varieties by near-infrared spectroscopy can better understand the organization structure and composition of meat, and give a theoretical explanation of meat variety identification from the point of view of molecule and radical group. Meanwhile, a large amount of redundant information in full spectrum wavelength data can be eliminated, improve the modeling efficiency, find the key ingredients indicator for meat variety identification. Thus, the near-infrared identification method for meat varieties was simplified, and it could greatly reduce the complexity of the identification instrument exploitation.

\section{Acknowledgements}

This study was supported by the Natural Science Foundation of Hebei Province (C2016201092).

\section{References}

[1] C.J. Liu, K.X. Li: Meat Industry, (2007) No.1, p.12. (In Chinese)

[2] X.Y. Niu, L.M. Shao: Advances in Computer Science Research, Vol. 83 (2018), p.84.

[3] I. Noda: Applied Spectroscopy, Vol. 47 (1993) No.9, p.1329.

[4] I. Noda: Vibrational Spectroscopy, Vol. 36 (2004) No.2, p.143.

[5] Z.H. You, H.M. Hong, F. Chen, et al.: Spectroscopy and Spectral Analysis, Vol. 38 (2018) No.5, p.1626. (In Chinese)

[6] S. Morita, Y. Ozaki: Chemometrics and Intelligent Laboratory Systems, Vol. 168 (2017), p.114.

[7] Y. Ozaki, K. Murayama and Y. Wang: Vibrational Spectroscopy, Vol. 20(1999) No. 2, p. 127.

[8] Y.L. Liu, Y.R. Chen, Y. Ozaki: Journal of Agricultural and Food Chemistry, Vol. 48 (2000) No. 3, p. 901.

[9] Y.L. Liu and Y.R. Chen: Meat Science, Vol. 57 (2001) No. 3, p.299. 
[10]H. Liu, B.R Xiang, L.B. Qu, et al.: Journal of China Pharmaceutical University, Vol. 37 (2006) No. 2, p.181. (In Chinese)

[11]J. Lu, B.R. Xiang and H. Liu: Progress in Pharmaceutical Sciences, Vol. 31 (2007) No. 7, p.303. (In Chinese)

[12]B. Chen, L. Cui, Z.X. Lin, et al.: Applied Chemical Industry, Vol. 45 (2016) No. 4, p.784. (In Chinese)

[13]X.Y. Niu, L.M. Shao, F. Dong, et al.: Spectroscopy and Spectral Analysis, Vol. 34 (2014) No.10, p.2737. (In Chinese)

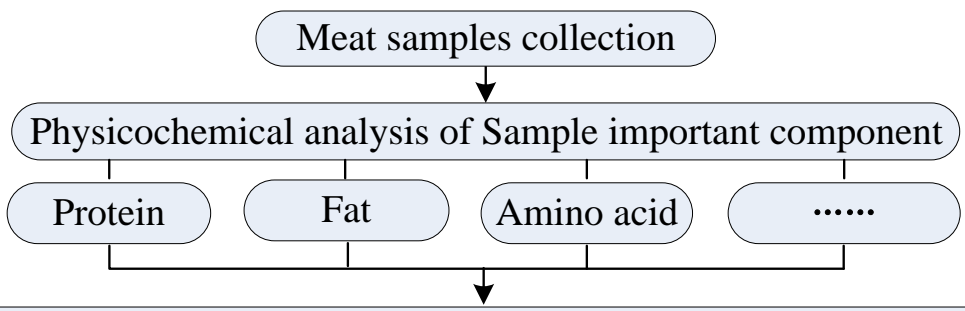

Key ingredients indicator selecting analysis of donkey meat identification

Selection of the corresponding structural radical group and absorption wavelength

Two-dimensional correlation near infrared spectroscopy of donkey meat samples under different perturbations

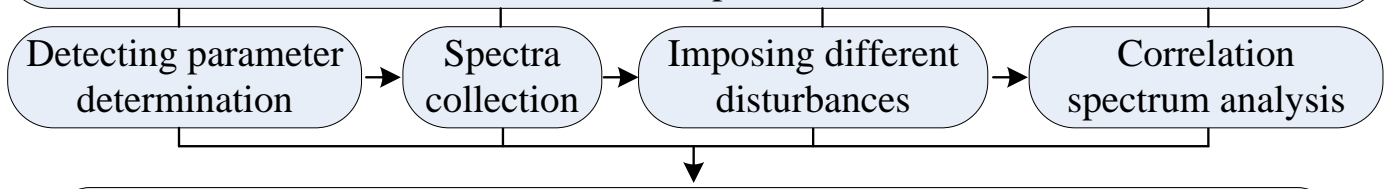

Further selecting the key ingredients, radical groups and absorption wavelength

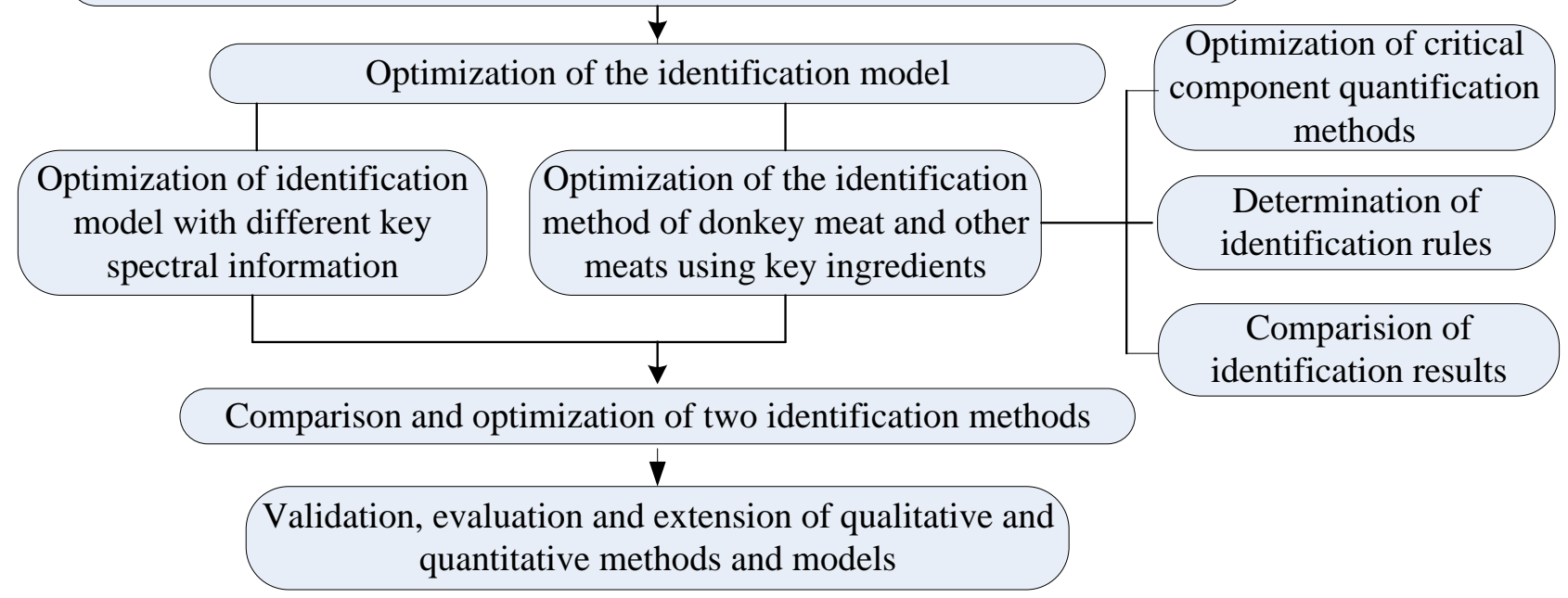

Figure 1. Research technical route of the two-dimensional near-infrared spectroscopy applied to the donkey meat identification 